\title{
Live vaccination tactics: possible approaches for controlling visceral leishmaniasis
}

\section{Noushin Saljoughian, Tahareh Taheri and Sima Rafati *}

Molecular Immunology and Vaccine Research Laboratory, Pasteur Institute of Iran, Tehran, Iran

Edited by:

Nahid Ali, Indian Institute of Chemical

Biology, India

\section{Reviewed by:}

Urszula Krzych, Walter Reed Army

Institute of Research, USA

Hira Nakhasi, Center for Biologics

Evaluation Research and Review, USA

\section{*Correspondence:}

Sima Rafati, Molecular Immunology and Vaccine Research Laboratory,

Pasteur Institute of Iran, 69 Pasteur

Avenue, Tehran 13164, Iran

e-mail: s_rafati@yahoo.com, sima-rafatisy@pasteur.ac.ir
Vaccination with durable immunity is the main goal and fundamental to control leishmaniasis. To stimulate the immune response, small numbers of parasites are necessary to be presented in the mammalian host. Similar to natural course of infection, strategy using live vaccine is more attractive when compared to other approaches. Live vaccines present the whole spectrum of antigens to the host immune system in the absence of any adjuvant. Leishmanization was the first effort for live vaccination and currently used in a few countries against cutaneous leishmaniasis, in spite of their obstacle and safety. Then, live attenuated vaccines developed with similar promotion of creating long-term immunity in the host with lower side effect. Different examples of attenuated strains are generated through long-term in vitro culturing, culturing under drug pressure, temperature sensitivity, and chemical mutagenesis, but none is safe enough and their revision to virulent form is possible. Attenuation through genetic manipulation and disruption of virulence factors or essential enzymes for intracellular survival are among other approaches that are intensively under study. Other designs to develop live vaccines for visceral form of leishmaniasis are utilization of live avirulent microorganisms such as Lactococcus lactis, Salmonella enterica, and Leishmania tarentolae called as vectored vaccine. Apparently, these vaccines are intrinsically safer and can harbor the candidate antigens in their genome through different genetic manipulation and create more potential to control Leishmania parasite as an intracellular pathogen.

Keywords: Leishmania, visceral leishmaniasis, live vaccine, live attenuated vaccines, live non-attenuated vaccines

\section{INTRODUCTION}

Several species of the protozoan genus Leishmania $(L)$ causes a group of parasitic diseases called Leishmaniasis which generates different clinical symptoms from cutaneous (CL) to visceral leishmaniasis (VL). People living in Latin America, the Middle East, parts of Africa, Asia, and India have been affected by VL (also named Kala azar) which is a very deadly disease caused mainly by $L$. (d) infantum, $L$. (d) donovani, and $L$. (d) chagasi species. Kala azar causes a clinical syndrome identified by repetitive fever, anemia, hepatosplenomegaly, and a wasting disease accompanied with muscular atrophy and finally leads to death after all the sufferings. Sand flies that have already bitten infected dogs or humans transfer parasites to other humans through their bites. These Leishmania parasites have numerous survival strategies among which the intracellular replication is the most famous one and prevents the parasites from direct contact to the immune system by the surrounding host cells.

A Th1 type cytokine milieu causes the parasite load to clear while a Th2 type leads to the host's susceptibility. Th1 cytokines can trigger macrophages, which are the major cells to destroy Leishmania parasites. To clear intracellular parasites, Th2 cells do not suffice since they induce a humoral response which has little or no effect on the parasites. Nowadays, controlling the disease depends mainly on chemotherapy as prophylactic or therapeutic vaccines are unavailable. VL chemotherapies have certain disadvantages such as the lengthy treatment time, costly drugs, and teratogenic effects. The reason for concern about resistance emergence is the long half-life of the chemotherapeutics (1-3).

The complex life cycle of Leishmania parasites, which consists of stages in animal or human and the sand fly vector, makes vaccine development more challenging (Figure 1A). An ideal antileishmanial vaccine should be able to solve current problems and limitations of other existing vaccines. As shown in Figure 1B, it should be safe, stable, reproducible, less risky, easily administered, stored and delivered, not reversible to infectious state, and able to induce long-term immunological memory and humoral and cellular responses.

In CL form of disease, the life-long protection is generated against the same disease and this is the fact that promises the feasibility of a vaccine. Deliberate infection with parasites at hidden body sites where scars ensue is a method that has been exploited in the leishmanization (LZ) practices of the last century (4). Nations, particularly in the Middle East, have successfully used the strategy for mass prevention of CL, but it need to improve due to persistence of monthly adverse effects and local lesions in $2-3 \%$ of cases (5).

In the late 1930s, researchers in Brazil showed that killed parasites were efficient when used as therapeutic as well as prophylactic; afterward first generation vaccines were produced from the whole killed Leishmania parasites (6). For many years, these vaccines 


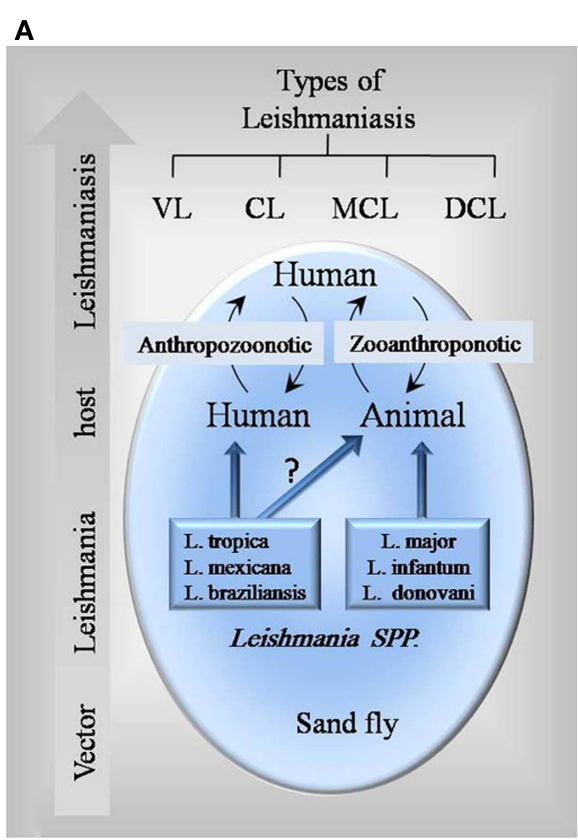

FIGURE 1 | Schematic figure of Leishmania interplay and different factors to consider in vaccine design. (A) Most of Leishmania parasites such as $L$. infantum and $L$. donovani are known to have both human and animal hosts, so preventive vaccines could be designed for

were tested either alone or combined with different adjuvants. So far, killed parasites had no enough efficacy as a potent vaccine to prevent disease, although they have demonstrated well-tolerated safety profiles (7).

First generation vaccines produced from VL Leishmania species have had no chance to be tested in clinical trials, since most vaccine studies have concentrated on CL. What have been included for the progression of Leishmania second generation vaccines are recombinant proteins, poly-proteins, DNA vaccines, and combinations thereof. In experimental infection systems, not only defined single molecules, but also multi-component vaccines have shown protection against VL. Coler et al. worked on LEISH-F1 + MPL-SE, which consisted of three recombinant Leishmania poly-protein (TSA-LmSTI1-LeIF), in association with monophosphoryl lipid and squalene as adjuvants (MPL-SE) (8). The synthetic RAPSODI $^{1}$ and two other DNA vaccines are in preclinical trials in Europe; one is being developed based on a viral vector by Paul Kaye (York University, UK) and another, LEISHDNAVAX ${ }^{2}$, by Mologen (Berlin, Germany) using a new technology named minimalistic immunogenically defined gene expression (MIDGE) to deliver selected Leishmania antigens; the latter can be used either solely or accompaniment to a synthetic adjuvant - double stem loop immunomodulator (dSLIM).

It is believed that if a candidate vaccine could stimulate immune system more similar to the natural disease, we will have a more

\footnotetext{
${ }^{1}$ http://www.fp7-rapsodi.eu/

${ }^{2}$ http://www.leishdnavax.org/
}

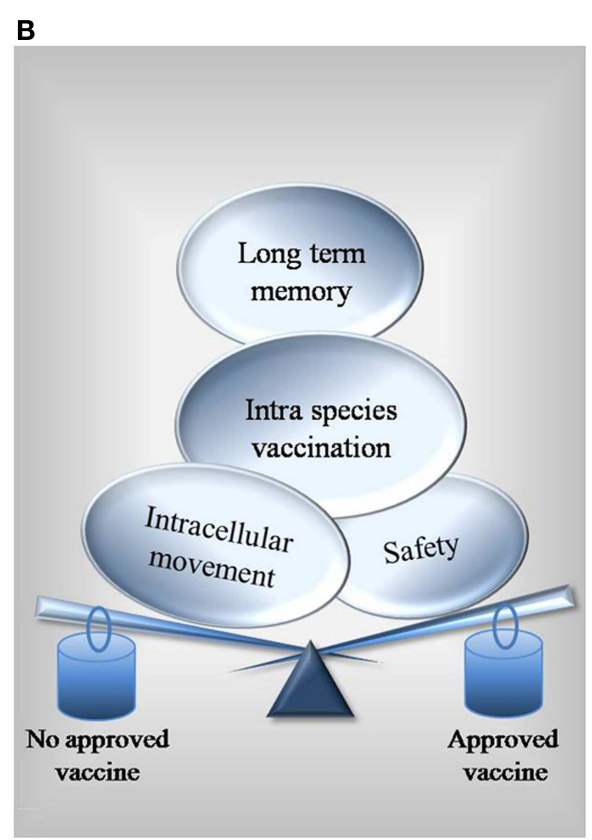

both. However, there is no identified reservoir animal host for some species like $L$. tropica. For these species, specific vaccines for human are needed. (B) Balance between different factors leads a vaccine to get approved.

efficient immune response. As the success of smallpox, measles, mumps, and rubella vaccines indicate that live attenuated vaccines are the touchstone for protection against their specific causing pathogen. As shown in Figure 2, different approaches were used based on whole parasite vaccine ranging from live active Leishmania vaccine (LZ) to live non-pathogenic vaccines.

Some attenuated strains were also developed through different approaches such as physical, chemical, and genetically attenuation. Much interest has been arisen in the development of genetically attenuated parasite vaccines due to the knowledge obtained in potential parasite virulence factors and the increased understanding of the antigens participating in immunity acquisition. Targeting and deleting genes that encode virulence factor genes essential for intracellular survival is the major general approach toward genetic attenuation of Leishmania parasites. Recently, there are few successful reports about live attenuated Plasmodium through genetical modification that can elicit long-lasting memory protection by producing antibodies and cellular immune responses (9). Interestingly, in recent human clinical trial using Plasmodium falciparum genetically attenuated parasites (PfGAP) as vaccine on volunteers showed the first in human proof of concept of this strategy that could inhibit the expansion of disease by decreasing the sporozoites (10).

Using BCG as a vaccine against Mycobacterium tuberculosis infection is a method which is comparable with utilizing nonpathogenic Leishmania species, such as a lizard parasite L. tarentolae, to develop live non-pathogenic parasites as VL vaccines. Although L. tarentolae can infect mammalian cells and change to amastigotes, it does not cause any disease or clinical symptoms 


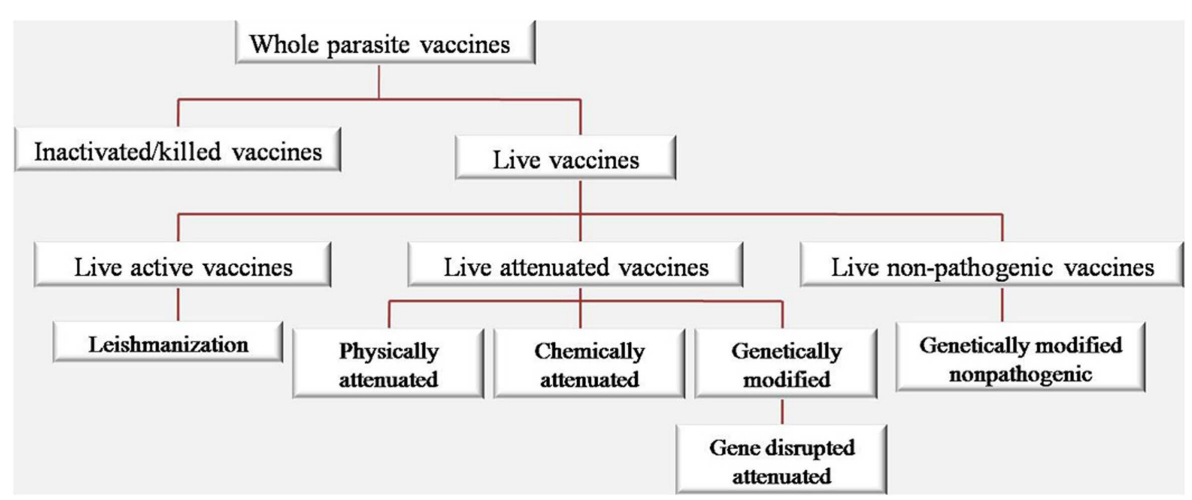

FIGURE 2 | Categorization of vaccine types based on whole Leishmania parasite.

in either mouse or hamster models $(11,12)$. Furthermore, due to general feasibility of human vaccination with live Salmonella and Lactococcus expressing exogenous antigens, they could serve another means to develop vaccine against leishmaniasis.

In this review, we have limited our scope to all types of live vaccinations against leishmaniasis and have considered them as vaccine candidates against leishmaniasis.

\section{LEISHMANIZATION (LIVE ACTIVE VACCINES)}

In the past, mothers used to expose their children's arms to be bitten by sand flies because they knew by experience that this would protect them from the severe disease in future. LZ was accepted in Israel and Russia after a method for axenic culture of the parasites was established (13). Using LZ was stopped because of HIV spreading, the use of immunosuppressive drugs, ethical reasons, uncontrolled permanent skin lesions, parasite persistence, and the inoculum quality control problems. The only usage of LZ at the present time is found in one of the endemic country, Uzbekistan, which is licensed and in Iran its efficacy is in human trials. Scientists are trying to improve the safety of this practice because it is the only way against Leishmania that has proved efficient in humans. The severity of primary lesions is reduced and wound healing is accelerated by including killed parasites in the inoculums and using adjuvants that improve quick immune responses $(14,15)$.

\section{LIVE ATTENUATED VACCINES}

Different methods such as physical attenuation: long-term vitro cultures (16), temperature sensitivity (17), $\gamma$-attenuation (18), and chemical attenuation: chemical mutagenesis (19), and parasite culture under drug pressure (20) were used to develop attenuated strains.

Instead, using a targeted gene disruption strategy can lead to a genetic alteration of the Leishmania genome that could help identifying essential genes for survival and/or virulence (2127) (Table 1). Generally speaking, live attenuated organisms are quite acceptable for vaccination because, first, such vaccines render native antigen into cells and improve activation of antigenpresenting cells at the same time by imitating the natural course of infection, which will lead to an optimal polarization of $\mathrm{CD} 4^{+} \mathrm{T}$ cells (28); second, the memory repertoire of the immune system is increased since a collection of complete antigens is delivered (in comparison with subunit-defined vaccines); and third, they assure antigen persistency by generating prolonged sub-clinical infection. Then, generation of antigen-specific effector and memory cells which react soon after infection may be allowed (29). Substantial protection in murine models against challenge has been conferred by attenuated strains, but potential for reversion is possible for ever, which makes them inappropriate for use in human vaccination. Actually, risk of subsequent reactivation, especially in HIV/Leishmania co-infection, is raised by the persistence of asymptomatic Leishmania infections. In addition, a loss of effectiveness for protective immunity can be resulted from physical and chemical attenuation, either because a sub-clinical infection cannot be formed by such strains or because they do not express critical antigen epitopes anymore (30). Although the experimental results have been promising so far, there are still some safety points that need to be considered in relation to the use of genetically attenuated parasites as vaccines. Prolonged immunity after re-infection induces live attenuated vaccines through maintaining a low level asymptomatic infection. Since the persistence of antigen is essential to generate effective memory responses to Leishmania, the establishment of sub-clinical infection is considered quite valuable. Patients who are immunocompromised (e.g., after HIV infection) have shown reactivation of Leishmania. This is the reason why it is necessary that the safety of attenuated parasites that cause a sub-clinical infection should be carefully investigated.

\section{LIVE PHYSICALLY ATTENUATED VACCINES}

It was shown by Mitchell et al. that long-term cultured promastigotes of L. major and L. tropica isolates could not cause lesions after cutaneous injection to mice (16). One year later, the effect of long-term cultivation of $L$. donovani promastigotes on cultured mouse and hamster macrophages in vitro was evaluated by Nolan et al. In a period of 48 days, the number of amastigotes derived from long-term promastigote cultures decreased only slightly in mice but rapidly in hamsters (46). In another experiment, 8 weeks after infection, long-term cultured L. amazonensis promastigotes induced smaller lesions, produced higher IFN- $\gamma$, and made smaller parasite load compared to the short-term cultured counterparts. Macrophages infected by long-term cultured parasites expressed 
Table 1 | Live attenuated vaccines against leishmaniasis.

\begin{tabular}{|c|c|c|c|c|}
\hline Attenuated vaccine form & Species & Animal model & Result & Reference \\
\hline \multicolumn{5}{|l|}{ PHYSICALLY ATTENUATED } \\
\hline \multirow[t]{5}{*}{ Long-term cultured } & L. major & C57BL/6 and & C57BL/: completely resistant; BALB/c: partially & $(16)$ \\
\hline & L. tropica & $\mathrm{BALB} / \mathrm{c}$ & protection, persistent low-grade cutaneous disease & \\
\hline & L. major & $\mathrm{BALB} / \mathrm{C}$ & Protection & $(31)$ \\
\hline & L. chagasi & $\mathrm{BALB} / \mathrm{c}$ & No protection & $(30)$ \\
\hline & L. amazonensis & C57BL/6 & Smaller lesions, $\uparrow$ IFN- $\gamma, \downarrow$ parasite load & $(32)$ \\
\hline Temperature sensitivity & L. braziliensis & $\mathrm{BALB} / \mathrm{c}$ & Protection & $(17)$ \\
\hline Radio-attenuated & L. major & CBA & Resistance to subsequent infection with L. mexicana & (33) \\
\hline Gamma irradiation & L. major & $\mathrm{CBA}$ and $\mathrm{BALB} / \mathrm{C}$ & Protection against homologs and heterologous challenge & $(18)$ \\
\hline \multicolumn{5}{|l|}{ CHEMICALLY ATTENUATED } \\
\hline $\begin{array}{l}\text { With } N \text {-methyl- } N^{\prime} \text {-nitro- } N \text { - } \\
\text { nitrosoguanidine }\end{array}$ & $\begin{array}{l}\text { Avirulent } / \mathrm{pg}^{-} \\
\text {deficient L. major }\end{array}$ & $\mathrm{BALB} / \mathrm{c}$ & $\downarrow$ Lesion size, resistance to a subsequent challenge & $(19)$ \\
\hline $\begin{array}{l}\text { Culturing in vitro under } \\
\text { gentamicin pressure }\end{array}$ & $\begin{array}{l}\text { L. mexicana and } \\
\text { L. major }\end{array}$ & $\mathrm{BALB} / \mathrm{c}$ & $\begin{array}{l}\text { No lesions, Th1-like responses } \\
\downarrow \text { Th2 responses, modulate the host immune response } \\
\text { Significant protection }\end{array}$ & $(20,34,35)$ \\
\hline $\begin{array}{l}\text { Culturing in vitro under } \\
\text { gentamicin pressure }\end{array}$ & L. infantum & Dogs & $\begin{array}{l}\text { No clinicopathological abnormalities } \\
\uparrow \text { IFN- } \gamma, \downarrow \text { IL-10, } \uparrow \text { IgG2 } \\
\uparrow \text { CD4+ and CD8+T cells }\end{array}$ & $(36-38)$ \\
\hline \multicolumn{5}{|l|}{ GENETICALLY ATTENUATED } \\
\hline dhfr-ts Null mutant & L. major & $\mathrm{BALB} / \mathrm{c}$ & Protective & $(21)$ \\
\hline $\begin{array}{l}\text { Cysteine proteinase-deficient } \\
\text { mutant }\end{array}$ & L. mexicana & $\begin{array}{l}\text { BALB/C, C57BL/6, } \\
\text { CBA/Ca }\end{array}$ & Immune response modulation, Th1 response & $(24)$ \\
\hline dhfr-ts Null mutant & L. major & Monkeys & No protection & (39) \\
\hline lpg2- & L. major & $\mathrm{BALB} / \mathrm{c}$ & Protection, no strong Th1 response & $(26)$ \\
\hline $\begin{array}{l}\text { Cysteine proteinase-deficient } \\
\text { mutants }\end{array}$ & L. mexicana & Hamsters & $\begin{array}{l}\text { Delayed disease onset } \\
\downarrow \text { Smaller lesions } \\
\downarrow \text { Parasite burden, } \downarrow \text { IL-10 and TGF-beta, and protection }\end{array}$ & $(40)$ \\
\hline LiSIR2( \pm ) mutant & L. infantum & $\mathrm{BALB} / \mathrm{c}$ & $\uparrow \mathrm{IFN}-\gamma / \mathrm{IL}-10$ ratio, $\uparrow \mathrm{NO}$, protection & $(27)$ \\
\hline $\begin{array}{l}\text { Phosphomannomutase-deficient } \\
\text { mutant }\end{array}$ & L. major & $\mathrm{BALB} / \mathrm{c}$ & $\begin{array}{l}\downarrow \mathrm{IL}-10 \text { and IL-13, } \uparrow \text { CD44hi T cell recruitment } \\
\text { Protection }\end{array}$ & $(41)$ \\
\hline LdCen1(-/-) mutant & L. donovani & $\begin{array}{l}\text { BALB/c SCID } \\
\text { hamsters }\end{array}$ & $\begin{array}{l}\uparrow I F N-\gamma, \text { IL-2, TNF, } \uparrow \text { IgG2a, } \uparrow \text { IFN- } \gamma / \mathrm{IL}-10 \text { ratio, } \uparrow \mathrm{NO}, \mathrm{Th} 1 \\
\text { response, long-lasting protection in hamsters }\end{array}$ & $(42)$ \\
\hline HSP70-II null mutant & L. infantum & $\mathrm{BALB} / \mathrm{c}$ & $\uparrow$ NO, type 1 responses & $(43)$ \\
\hline Ldp27(-/-) mutant & L. donovani & $\mathrm{BALB} / \mathrm{c}$ & Long-term protection & $(44)$ \\
\hline $\left.\mathrm{cLdCen}^{-/-}\right)$mutant & L. donovani & Dogs & $\begin{array}{l}\uparrow \text { Type } 1, \downarrow \text { Type } 2 \\
\uparrow \text { Immunogenicity }\end{array}$ & $(45)$ \\
\hline
\end{tabular}

high level of chemokine CXCL10 mRNA, which might activate these cells to kill the parasites (32). Nevertheless, there are several similar trials which led to ineffectiveness, such as long-term in vitro culture of L. chagasi that did not create protective immunity (30). Using temperature-sensitive avirulent parasite clones, the immunized susceptible BALB/c mice were successfully protected against L. braziliensis (17). Radio-attenuation, first introduced in 1974 by Lemma et al., is another physical approach for preparation of Leishmania vaccine (47). The resistance of CBA mice to subsequent infection with L. mexicana is highly increased by administration of radio-attenuated L. major vaccines (33). In another experiment, gamma irradiation of L. major elicited a high degree of protection against homologs and heterologous challenge in CBA and BALB/c mice (18). Although most of these methods showed promising protective effects, they were not further used in research studies of vaccination against Leishmania species, due to safety issues regarding incomplete inactivation and reversion of infectivity (Table 2). 
Table 2 | Properties of different types of live vaccines based on whole organisms.

\begin{tabular}{|c|c|c|}
\hline Type of live vaccines & Benefits & Concerns \\
\hline $\begin{array}{l}\text { Live non-attenuated } \\
\text { vaccines }\end{array}$ & Almost successful and immunity $(48,50)$ & $\begin{array}{l}\text { Exacerbate the disease, reversion to virulence, large persistent lesions, } \\
\text { psoriasis, and immunosuppression } \\
\text { Not reproducible }(48) \text {, no efficacy, and no standardization and quality } \\
\text { control }(48,50) \\
\text { Risk of HIV transmission }\end{array}$ \\
\hline Chemically attenuated & Cheaper & $\begin{array}{l}\text { No safety, high risk, incomplete attenuation, no efficacy, not reproducible, } \\
\text { risk of random mutations, non-specific attenuation (51), and reversion to } \\
\text { virulence (51) }\end{array}$ \\
\hline $\begin{array}{l}\text { Non-pathogenic } \\
\text { organism }\end{array}$ & $\begin{array}{l}\text { Safer (52), cross-reactivity between species (48), } \\
\text { induce both humoral and cellular response (48) } \\
\text { Lower risk of reversion to the virulent phenotype, } \\
\text { highly immunogenic } \\
\text { Natural course of infection } \\
\text { For some easy administration }\end{array}$ & $\begin{array}{l}\text { Not appealing prospect (48) } \\
\text { Possible reversion to virulence or reactivation (52) } \\
\text { Presence of antibiotic resistance genes (52) } \\
\text { Storage and delivery }\end{array}$ \\
\hline
\end{tabular}

\section{LIVE CHEMICALLY ATTENUATED VACCINES}

To immunize susceptible BALB/c mice against challenge with virulent $L$. major, Kimsey et al. used an avirulent clone of $L$. major which was prepared after several in vitro treatments of a virulent population of $L$. major with the mutagen, $\mathrm{N}$-methyl$N^{\prime}$-nitro- $N$-nitrosoguanidine (MNNG), and could control lesion size in the challenge mice model (19). It has been shown that an avirulent lipophosphoglycan-deficient $L$. major clone is able to elicit resistance to a subsequent challenge with virulent $L$. major while it is unable to produce cutaneous lesions in susceptible $\mathrm{BALB} / \mathrm{c}$ mice (19). Similarly, in another experiment, avirulent lipophosphoglycan-deficient $L$. donovani parasites could not generate visceral infection in hamster model after inoculation through the intra cardiac route, contrary to virulent $L$. donovani (53). Different species of Leishmania have been attenuated by culturing in vitro under gentamicin pressure successfully such as $L$. mexicana, L. major, L. infantum, and L. donovani. While wild-type (WT) parasites survived and multiplied, the attenuated strains were able to invade but they neither could survive within bone marrowderived macrophages in vitro nor induce cutaneous lesions in $\mathrm{BALB} / \mathrm{c}$ mice after about 12 weeks. High level of protection was induced in mice against challenge with WT parasites by both attenuated lines of L. mexicana and L. major (20). This was accompanied by a $\mathrm{CD} 4^{+}$Th1-like response in $\mathrm{BALB} / \mathrm{c}$ mice that was shown by the cytokine profile of their WT L. mexicana promastigotesstimulated splenocytes (34). Growth of the WT parasites was excessively controlled in experiments wherein mice were simultaneously inoculated (either at the same site or on separate sites) with attenuated and WT parasites, showing that the attenuated parasites have a possible therapeutic role. Comparing dogs infected with either WT L. infantum or gentamicin-attenuated L. infantum $\mathrm{H}$-line, no pathological abnormalities were observed in the latter group, which induced significantly higher IFN- $\gamma$ and lower IL-10 levels with the highest levels of IgG2 subclass in their sera (37). Also, proliferation of mononuclear cells is associated with cellular immunity in immunized dogs (38). However, in addition to the difficulty of large-scale production of these physically attenuated vaccines and their delivery to the field in appropriate conditions, the major drawback is their loss of effectiveness for protective immunity due to their inability to form sub-clinical infection and express critical antigen epitopes (30) (Table 2).

\section{LIVE GENETICALLY ATTENUATED VACCINES}

Development of transfection technology has acted as a powerful reverse molecular genetics tool for genetic modifications in the last two decades. Gene delivery into such unicellular pathogens as Leishmania has created a great revolution in making genetically defined vaccines through knocking out/in certain genes. DNA delivery by physical methods is a very efficient and easy system; DNA fragments are best transferred into parasites nuclei by transfection through electroporation (54). A linearized construct containing antibiotic resistant genes should be integrated into the genome through homologous recombination (HR) to remove a gene. This allows a DNA sequence transfer into the locus of interest in the Leishmania genome using two flanking sequences in both sides of the gene (54).

To generate an absolute knockout, the Leishmania parasite needs a second construct to bear another antibiotic resistant gene to replace the second gene alleles. The cell phenotype is altered by this manipulation and new parasite features are naturally 
transferred to the next generations through inheritance. Controlling the gene in its new genome location is the most crucial concern in gene targeting because it may affect the normal gene functionality in both sides of the target. Therefore, gene entrance location is very important and should be confirmed by molecular genetics methods although Leishmania genome is relatively easy to manipulate. Phenotypic changes (e.g., morphology, growth, infectivity) of the manipulated parasite after each transfection are other critical issues that need to be studied.

In this direction, one of the first experiments to vaccinate mice against challenge with virulent $L$. major was done by Titus et al. (21) using $d h f r$-ts null mutant of L. major obtained by gene targeting. Although it could not produce protective immunity in primates and needed further improvement for vaccine application (39), it could elicit considerable resistance phenotype after $\mathrm{BALB} / \mathrm{c}$ mice challenge with virulent L. major (21). L. mexicana mutants lacking cysteine proteinase genes generated by targeted gene disruption were tested on murine and hamster models in another attempt and could induce delayed disease onset, smaller lesions, and lower parasite burden in mice and hamsters $(24,40)$. Thus, the idea of the feasibility of using genetically attenuated live Leishmania to achieve protective immunity was supported by such findings. Uzonna et al. showed that highly susceptible mice could be protected against virulent challenge without inducing a strong Th1 response when vaccinated with phosphoglycan-deficient $L$. major (26). A much less capacity compared to the WT parasites was shown in L. donovani BT1 null mutant for inducing infection in mice, and those susceptible to infection against $L$. donovani challenge attained protective immunity (25). Silvestre et al. showed that SIR2-deficient (silent information regulatory 2) L. infantum induced a clear IFN- $\gamma /$ IL-10 pattern that is associated with protection patterns (27). In another study, susceptible $\mathrm{BALB} / \mathrm{c}$ mice showed protection against infection when vaccinated with avirulent $L$. major phosphomannomutase-deficient parasites (41). Kedzierski et al. concluded that the factors that play essential parts in eliciting protection against Leishmania are increase in the number of T cells, their rapid recruitment to lymph nodes upon infection, and lower production of IL-13 and IL-10 (which leads to high IFN- $\gamma / \mathrm{IL}-10$ ratio). It was shown in 2009 that live attenuated $L$. donovani parasites by gene disruption of centrin gene (LdCen $1^{-l-}$ ) could be live, safe, and induce protection in susceptible BALB/c mice, immunocompromised severe combined immunodeficiency (SCID) mice and hamsters. Infection with L. braziliensis, which causes mucocutaneous leishmaniasis, could be prevented if mice were immunized with $\operatorname{LdCen} 1^{-1-}$ (42). It was shown by Fiuza et al. that strong antibody production, Type 1 polarization, and Type 2 inhibition could be induced by $\mathrm{LdCen}^{-/-}$vaccine in dogs, as an important reservoir host (45). Dey et al. have shown that $L$. donovani mutant of amastigote-specific protein p27 knockout $\left(\operatorname{Ldp} 27^{-1-}\right)$ as live attenuated parasites are safe, induce protective immunity, and can provide protection against homologous and heterologous Leishmania species (44). Carrion et al. believe that the ability of a safe genetically modified $L$. infantum mutant, which lacks both HSP70-II alleles ( $\triangle$ HSP70-II), provide protection against L. major infection in $\mathrm{BALB} / \mathrm{c}$ and can lead to the production of high levels of NO, type 1 immune responses, and IgG subclass analyses in mice (43). However, there are some limitations for their extensive use such as safety constraints due to reversion to virulent form especially in immunosuppressed individuals and manufacturing concerns.

\section{LIVE NON-PATHOGENIC VACCINES}

Utilization of non-pathogenic species as Salmonella enteric, Lactococcus lactis, and L. tarentolae to develop live attenuated parasites as $\mathrm{VL}$ vaccines is another approach. This approach has shown enhanced antigen presentation and potent Th1 response similar to BCG, a successful vaccine against $M$. tuberculosis infection (Table 3). These methods can be further refined through the use of their recombinants expressing antigens of virulent Leishmania spp. In general, the most promising strategic alternative against VL can be claimed to be the use of live, non-pathogenic/genetically engineered strains of these species.

\section{SALMONELLA ENTERICA}

Salmonella $(S)$ are intracellular pathogens that upon entrance to human macrophages induce a viscerotropic immune response similar to Leishmania. Development of live Salmonella vaccines as a method for delivering heterologous antigens was discussed for the first time in 1987 (66). The important advantage of using attenuated Salmonella for vaccination against VL is their low production cost, storage at room temperature, and their oral, needle-free application if rehydrated. Since orally administered live attenuated Salmonella spp. that express heterologous antigens are safe and highly immunogenic, they are promising candidates; they can elicit prolonged, protective, systemic, and mucosal immune responses against the heterologous pathogen (67).

In vivo inducible promoters and optimized expression systems are used to construct novel attenuated Salmonella vaccines that deliver antigens and show a host protective effect in small rodent models of VL. Live Salmonella needs more studies to promote their further application.

Furthermore, for delivery and expression of vaccine antigens in the host, several attenuated lines of S. typhimurium have been generated. For more safety, more than one attenuating mutation can be incorporated in a vaccine. Several derived antigens (target carbohydrate, protein) or epitopes from different pathogens, viruses, bacteria, and eukaryotic parasites are expressed by combined Salmonella vaccines in the form of capsules, fimbria, or flagellum, either within or on the surface of the cell (68). A very significant resistance was developed against a $L$. major challenge infection by the mice that had been orally immunized with gp63transformed S. typhimurium $(55,56)$. S. typhimurium derivatives (GIDMIF, GIDIL2, GIDIFN, and GIDTNF) expressed cytokines in vitro under anaerobic conditions. They were stably colonized in orally immunized BALB/c mice more than 14 days and showed protective effect which correlated with the induction of inducible nitric oxide synthase (57).

Lange et al. showed that production of IFN- $\gamma$ could induce protection against $L$. major infection in susceptible BALB/c mice and were enhanced as a result of using LACK antigens in DNASalmonella primer-booster vaccination compared to that with the DNA alone (59). In a recent study, Schroeder et al. identified two novel candidate vaccine antigens (LinJ08.1190 and LinJ23.0410) 
Table 3 | Live non-pathogenic vaccines against leishmaniasis.

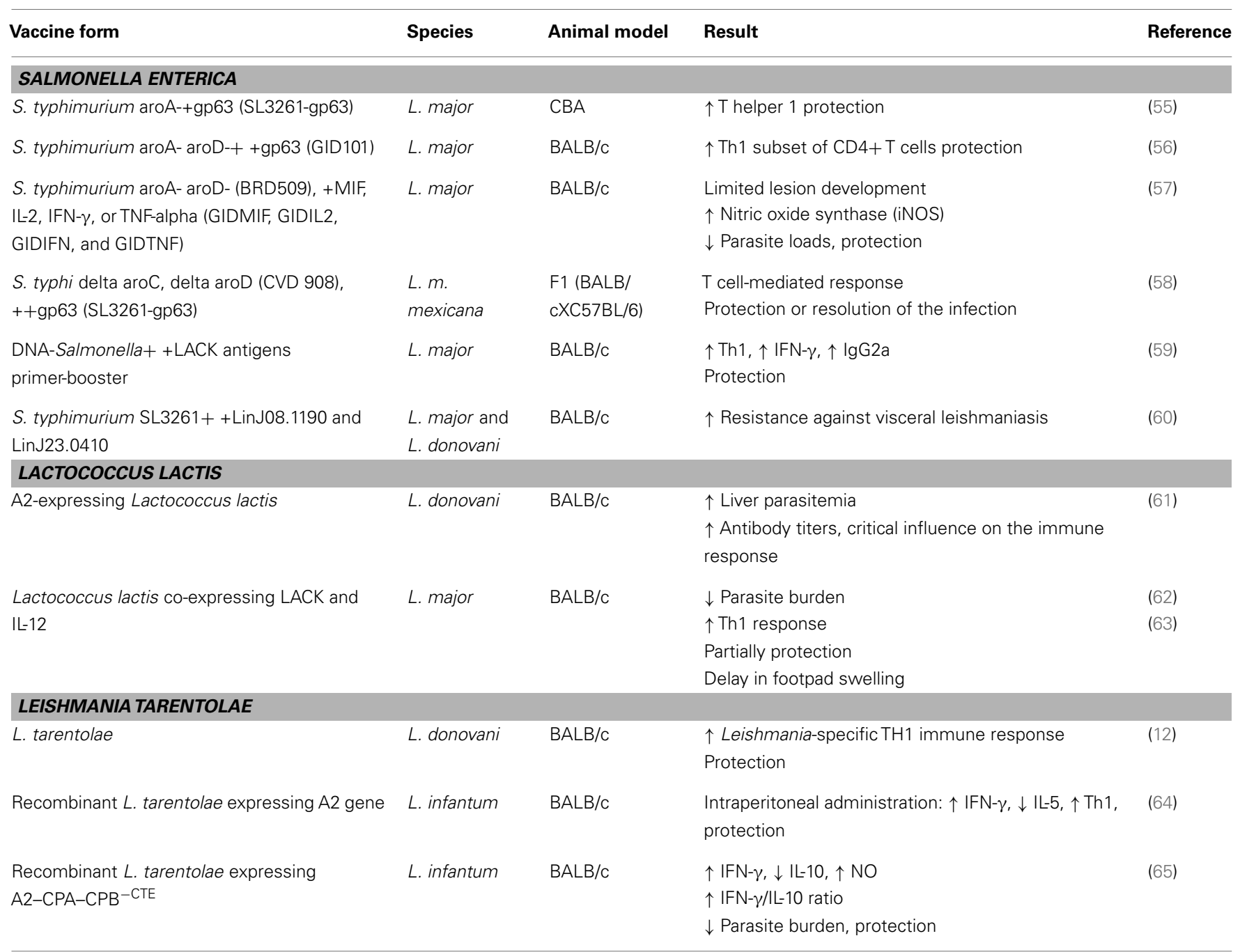

by reverse vaccinology and utilized them in the construction of live Salmonella carriers against VL, which reduced visceralization considerably and increased resistancy against $L$. donovani infection in susceptible BALB/c mice (60).

\section{LACTOCOCCUS LACTIS}

Lactococcus lactis is a Gram-positive, non-pathogenic, noncolonizing lactic acid bacterium (69), which is industrially important and is frequently used in the preparation of fermented foods and dairies; FDA has given it a generally recognized as safe (GRAS) status [(70); aminopeptidase enzyme preparation derived from $L$. lactis (21CFR184.1985)].

It has been used as a live bacterial delivery vector for more than 10 years (71) and scientists are being encouraged to use it as a live vaccine against leishmaniasis. A2-expressing L. lactis live vaccines have been generated and evaluated by Yam et al. against $L$. donovani in $\mathrm{BALB} / \mathrm{c}$ mice. This $\mathrm{A} 2$ anchored to the cell wall has a critical influence on the immune response; this subcellular location of antigen expression causes the highest reduction in liver parasitemia, induces the highest level of antigen-specific antibody titers which is seen at both low- and high-dose L. donovani parasite challenges (61). In another study of this group it was shown, using LACK- and IL-12-expressing L. lactis, that subcutaneous immunization against $L$. major infection delays footpad swelling, indicating the necessity for co-administration of L. lactis/sec IL-12 (secreting IL-12) as a Th1-inducing adjuvant (63). Again in another study, the same group showed that if live $L$. lactis secreting both LACK and IL-12 was used, oral immunization was the only regimen that could protect $\mathrm{BALB} / \mathrm{c}$ mice partially against L. major infection (62). The L. lactis line generated in these studies provides an attractive cornerstone for further research on live-based vaccines against leishmaniasis and other pathogens.

\section{LEISHMANIA TARENTOLAE}

Recently, the use of a non-pathogenic Leishmania vector (L. tarentolae) was suggested by Breton et al. (12) as a vaccine candidate against leishmaniasis which is known as non-pathogenic for human since it is not able to generate any manifestation of human leishmaniasis. Although this parasite is non-pathogenic in either 
mouse or hamster models because it lacks any clinical symptoms, it can infect mammalian cells and transform into amastigotes (72). Genome sequence analyses have revealed that this parasite is syntonic to the three sequenced pathogenic Leishmania species ( $L$. major, L. braziliensis, and L. infantum) and that more than $90 \%$ of the approximately 8200 genes are shared by all the species. Nevertheless, some of the essential genes that are relevant to pathogenicity in pathogenic strains or expressed in amastigote form are absent in $L$. tarentolae or were in variable copy number. This supports the idea that some of these genes are possible to be associated with reduction of pathogenic capacity in L. tarentolae and make it an intracellular parasite and its diminished pathogenic potential to humans. As an example, the amastin family, especially the delta group as just two copy number in L. tarentolae while high copy numbers (12-25) are found in the pathogenic species (73). Why L. tarentolae cannot replicate efficiently in mammalian macrophages can be explained by the absence of these proteins. It has been shown in experimental vaccine trials that a single intra peritoneal immunization of $L$. tarentolae elicited a protective immune response against $L$. donovani in susceptible BALB/c mice; it was concluded that it was a result of an enhanced antigen presentation and potent Th1 immune response (12). Since L. tarentolae is a safe vector for use as a vaccine, it can be more effective antiLeishmania vaccine by genetic manipulation in order to induce transgenic L. tarentolae which expresses certain immunodominant Leishmania antigens.

Effort has also been made to use L. tarentolae as a specific deliver and expression system for Leishmania antigens in host. The L. donovani A2 antigen was expressed in L. tarentolae, which normally lacks this protein (74) and used as a vaccine strain in an experimental mouse model. The susceptible mice were protected against $L$. infantum infection through vaccination following high levels of IFN- $\gamma$ were produced (64). In addition, L. tarentolae can be used as a promising live vaccine vector against intracellular pathogens. This idea was examined for the first time in an experiment using a recombinant $L$. tarentolae expressing HIV-1 Gag protein as a candidate HIV-1 vaccine. It was shown that the vaccine induces a strong cell-mediated immunity in BALB/c mice and decreases HIV-1 replication in an ex vivo condition (75). Also, a novel live vaccine using recombinant L. tarentolae expressing E7 protein for the protection of mice against HPV-associated tumors was produced and evaluated (76). It is worth mentioning that this vaccine showed the best protection and minimum tumor size among all other groups against TC-1-induced tumors (76).

Our team produced a recombinant $L$. tarentolae expressing the $\mathrm{A} 2-\mathrm{CPA}-\mathrm{CPB}^{-\mathrm{CTE}}$ tri-gene fusion that are three important vaccine candidate antigens of $L$. infantum, as a new live vaccination strategy against visceral form of leishmaniasis in twomodalities, namely DNA/live and live/live vaccination in BALB/c mice. We demonstrated how prime-boost (DNA/live) strategies using recombinant $L$. tarentolae-based vaccines elicited promising immunization against a high-dose virulent $L$. infantum challenge (65). We also tested live/live $L$. tarentolae-A2-CPA-CPB-CTE prime-boost vaccination regime in hamsters and showed that it represented an appropriate animal model in the discovery of potential antigens that could be used in the control of canine VL (unpublished data). The parasite loads in both visceral organs were controlled in the vaccinated hamsters reaching a negligible level by day 56 post challenge, demonstrating its strong vaccine potential. Five weeks after infection by $L$. infantum, hamsters that had received the live vaccine produced higher levels of anti- $L$. infantum lysate antibodies than those injected with PBS control.

In another attempt, we tested the efficacy of a novel combination of established protective parasite antigens expressed by L. tarentolae together with saliva antigens as a vaccine strategy against L. major infection. Different DNA/live and live/live primeboost vaccination modalities with live recombinant $L$. tarentolae stably expressing cysteine proteinases (type I and II, CPA/CPB) and PpSP15, an immunogenic salivary protein from Phlebotomus papatasi, a natural vector of $L$. major, were tested in both susceptible $\mathrm{BALB} / \mathrm{c}$ and resistant $\mathrm{C} 57 \mathrm{BL} / 6$ mice. In both strains of mice, the strongest protective effect was observed when priming with PpSP15DNA and boosting with PpSP15 DNA and live recombinant $L$. tarentolae stably expressing cysteine proteinase genes (accepted in PLoS NTD, 2014).

Regarding vaccine development in dogs, with lack of enough knowledge about canine leishmaniasis and canine immunity, it is almost impossible to predict the results obtained from the mouse and hamster models, if vaccine candidates can work in dogs. Therefore, it is essential to do more studies on dogs for both new vaccine candidates and immune response analyses. Whether or not protection will be achieved, results of such tests would be valuable for the advancement of knowledge about canine leishmaniasis and giving a guided direction to future protection strategies. It is worth to mention that our group is testing the genetically knock in L. tarentolae expressing the $\mathrm{A} 2-\mathrm{CPA}-\mathrm{CPB}^{-\mathrm{CTE}}$ tri-gene fusion as a live vaccination strategy with different modalities in outbreed dogs.

\section{CONCLUSION}

Unlike most other pathogens, Leishmania never clears fully by immune system and we do not need sterile immunity. The important issue for maintenance of immunity is believed to be the presence of small number of live parasite in the host. Live replicating parasites or just persistent antigens are believed to be important for the maintenance of effector memory like $\mathrm{T}$ cells but not for central memory $\mathrm{T}$ cells. It has been reported that the quality of memory cells in the presence and absence of live parasite are different in CL (77). In the case of VL, persistence of parasite antigen is important for generating antigen-specific effector $\mathrm{T}$ cells, although more depth studies are required to be analyzed in the case of non-pathogenic and/or genetically attenuated Leishmania parasite (44). During Leishmania infection, we need a methodical understanding of how the immunological memory is generated and maintained, what the sustained long-term protective immune responses are, and through what mechanisms vaccines stimulate protective immunity. An ideal anti-Leishmania vaccine must maintain constant turnover of Leishmania-specific memory cells in vaccinated host, otherwise repeated booster injections would be required (78).

Immune response to Leishmania is very complicated and for wisely designing vaccines we need to know which $\mathrm{T}$ cell determinants act as IFN- $\gamma$ inducer $(\mathrm{CD} 8+$ or $\mathrm{CD} 4+\mathrm{T}$ cell $)$ and are 
essential for long-term immunity. Long-lasting protective immunity induced by vaccination is a pragmatic goal for control of parasitic infections. In LZ, the only successful strategy that has been used to induce resistance to cutaneous leishmaniasis, after obviation of the infection, individuals are resistant to re-infection. It is now clear that in mice infected with WT parasites, heterogeneous memory CD4+ T cell pool contain two subsets, specified by their expression of the LN-homing molecule CD62L, one of them, effector memory $\mathrm{T}$ cells, has the characteristics of effector cells (CD62Llo) and the other one, central memory T cells, act as a repository of antigen-specific $\mathrm{T}$ cells (CD62Lhi) and can extend upon rechallenge, differentiate into effector $\mathrm{T}$ cells, and refill the effector cell population $(79,80)$. The latter which expressed CD62L and lodged to the lymph nodes, expand early after infection with $L$. major (81). However, the first population of cells CD62Llo effector $\mathrm{T}$ cells could intercede resistance faster than the CD62Lhi central memory $\mathrm{T}$ cells (80). In other words, at providing immunity to rechallenge in leishmaniasis central memory $\mathrm{CD} 4+\mathrm{T}$ cells that could be maintained without persistent parasites were less effective. This observation indicates that for immunity maintenance and providing long-term immunologic memory, persistent parasites may well be needed (82). Therefore, on this basis the idea of using live vaccine either in attenuated or non-pathogenic form is strengthened.

It is preferred that attenuating process of Leishmania strains for the production of live vaccine be done selectively (i.e., only in intracellular form or amastigotes); this will allow the cultivation of promastigotes in large-scale. Attenuation needs to be optimized so that the power of live parasite vaccines can be improved, but it should be noted that reversion of these parasites to the virulent form restricts their use. In other words, returning back to virulence is also probable; hence, the need for the production of new safer live vaccine vectors such as non-pathogenic $L$. tarentolae harboring immunogenic antigens that can enhance antigen presentation and elicit potent immune responses, without any risk of disease development in humans, becomes obvious. Using L. tarentolae as non-pathogenic vector is promised because of its safety and easy adaptation to mammalian system. Also, it has not the ability to revert to pathogenic form due to its non-pathogenic intrinsic property $(11,12)$. But what is certain is that $L$. tarentolae could not long survive in the mammalian cell, so it is best to think of some strategies to prolong its life there. Finally, there are still several obstacles for utilization of live non-pathogenic Leishmania, such as lyophilization and storage of this organism, which need special attention and serious research.

\section{ACKNOWLEDGMENTS}

We thank Amir Mizbani for critically reading and editing the manuscript. This work was supported by Pasteur Institute of Iran, Iran Ministry of Health, and National Science Foundation of Iran (grant number 91053539).

\section{REFERENCES}

1. Sundar S, Chatterjee M. Visceral leishmaniasis - current therapeutic modalities. Indian J Med Res (2013) 123(3):345-52.

2. Olliaro P, Darley S, Laxminarayan R, Sundar S. Cost-effectiveness projections of single and combination therapies for visceral leishmaniasis in Bihar, India. Trop Med Int Health (2009) 14:918-25. doi:10.1111/j.1365-3156.2009.02306.x
3. Olliaro P, Sundar S. Anthropometrically derived dosing and drug costing calculations for treating visceral leishmaniasis in Bihar, India. Trop Med Int Health (2009) 14:88-92. doi:10.1111/j.1365-3156.2008.02195.x

4. Nadim A, Javadian E, Tahvildar-Bidruni G, Ghorbani M. Effectiveness of leishmanization in the control of cutaneous leishmaniasis. Bull Soc Pathol Exot Filiales (1983) 76:377-83.

5. Hosseini SM, Hatam GR, Ardehali S. Characterization of Leishmania isolated from unhealed lesions caused by leishmanization. East Mediterr Health J (2005) 11:240-3.

6. Noazin S, Modabber F, Khamesipour A, Smith PG, Moulton LH, Nasseri K, et al. First generation leishmaniasis vaccines: a review of field efficacy trials. Vaccine (2008) 26:6759-67. doi:10.1016/j.vaccine.2008.09.085

7. Noazin S, Khamesipour A, Moulton LH, Tanner M, Nasseri K, Modabber F, et al. Efficacy of killed whole-parasite vaccines in the prevention of leishmaniasis: a meta-analysis. Vaccine (2009) 27:4747-53. doi:10.1016/j.vaccine.2009.05.084

8. Coler RN, Goto Y, Bogatzki L, Raman V, Reed SG. Leish-111f, a recombinant polyprotein vaccine that protects against visceral leishmaniasis by elicitation of CD4+ T cells. Infect Immun (2007) 75:4648-54. doi:10.1128/IAI.00394-07

9. Mueller A-K, Labaied M, Kappe SH, Matuschewski K. Genetically modified Plasmodium parasites as a protective experimental malaria vaccine. Nature (2005) 433:164-7. doi:10.1038/nature03188

10. Finney OC, Keitany GJ, Smithers H, Kaushansky A, Kappe S, Wang R. Immunization with genetically attenuated $P$. falciparum parasites induces long-lived antibodies that efficiently block hepatocyte invasion by sporozoites. Vaccine (2014). doi:10.1016/j.vaccine.2014.02.055

11. Wilson V, Southgate B. Lizard Leishmania. Biology of the Kinetoplastida. San Diego, CA: Academic Press (1978). p. 244-68.

12. Breton M, Tremblay MJ, Ouellette M, Papadopoulou B. Live nonpathogenic parasitic vector as a candidate vaccine against visceral leishmaniasis. Infect Immun (2005) 73:6372-82. doi:10.1128/IAI.73.10.6372-6382.2005

13. Koufman Z, Egoz N, Greenblatt CL, Handman E, Montilio B, Even-Paz Z. Observations on immunization against cutaneous leishmaniasis in Israel. Isr J Med Sci (1978) 14:218-22.

14. Khamesipour A, Dowlati Y, Asilian A, Hashemi-Fesharki R, Javadi A, Noazin $S$, et al. Leishmanization: use of an old method for evaluation of candidate vaccines against leishmaniasis. Vaccine (2005) 23:3642-8. doi:10.1016/j.vaccine. 2005.02.015

15. Tabbara KS, Peters NC, Afrin F, Mendez S, Bertholet S, BelkaidY, et al. Conditions influencing the efficacy of vaccination with live organisms against Leishmania major infection. Infect Immun (2005) 73:4714-22. doi:10.1128/IAI.73.8.47144722.2005

16. Mitchell GF, Handman E, Spithill TW. Vaccination against cutaneous leishmaniasis in mice using nonpathogenic cloned promastigotes of Leishmania major and importance of route of injection. Aust J Exp Biol Med Sci (1984) 62(Pt 2):145-53. doi:10.1038/icb.1984.14

17. Gorczynski RM. Immunization of susceptible BALB/c mice against Leishmania braziliensis. II. Use of temperature-sensitive avirulent clones of parasite for vaccination purposes. Cell Immunol (1985) 94:11-20. doi:10.1016/0008-8749(85) 90081-4

18. Rivier D, Shah R, Bovay P, Mauel J. Vaccine development against cutaneous leishmaniasis. Subcutaneous administration of radioattenuated parasites protects CBA mice against virulent Leishmania major challenge. Parasite Immunol (1993) 15:75-84. doi:10.1111/j.1365-3024.1993.tb00587.x

19. Kimsey PB, Theodos CM, Mitchen TK, Turco SJ, Titus RG. An avirulent lipophosphoglycan-deficient Leishmania major clone induces CD4+ T cells which protect susceptible BALB/c mice against infection with virulent $L$. major. Infect Immun (1993) 61:5205-13.

20. Daneshvar H, Coombs GH, Hagan P, Phillips RS. Leishmania mexicana and Leishmania major: attenuation of wild-type parasites and vaccination with the attenuated lines. J Infect Dis (2003) 187:1662-8. doi:10.1086/374783

21. Titus RG, Gueiros-Filho FJ, De Freitas LA, Beverley SM. Development of a safe live Leishmania vaccine line by gene replacement. Proc Natl Acad Sci U S A (1995) 92:10267-71. doi:10.1073/pnas.92.22.10267

22. Dumas C, Ouellette M, Tovar J, Cunningham ML, Fairlamb AH, Tamar S, et al. Disruption of the trypanothione reductase gene of Leishmania decreases its ability to survive oxidative stress in macrophages. EMBO J (1997) 16:2590-8. doi:10.1093/emboj/16.10.2590

23. Hubel A, Krobitsch S, Horauf A, Clos J. Leishmania major Hsp100 is required chiefly in the mammalian stage of the parasite. Mol Cell Biol (1997) 17:5987-95. 
24. Alexander J, Coombs GH, Mottram JC. Leishmania mexicana cysteine proteinase-deficient mutants have attenuated virulence for mice and potentiate a Th1 response. J Immunol (1998) 161:6794-801.

25. Papadopoulou B, Roy G, Breton M, Kundig C, Dumas C, Fillion I, et al. Reduced infectivity of a Leishmania donovani biopterin transporter genetic mutant and its use as an attenuated strain for vaccination. Infect Immun (2002) 70:62-8. doi:10.1128/IAI.70.1.62-68.2002

26. Uzonna JE, Spath GF, Beverley SM, Scott P. Vaccination with phosphoglycandeficient Leishmania major protects highly susceptible mice from virulent challenge without inducing a strong Th1 response. J Immunol (2004) 172:3793-7.

27. Silvestre R, Cordeiro-Da-Silva A, Santarem N, Vergnes B, Sereno D, Ouaissi A. SIR2-deficient Leishmania infantum induces a defined IFN-gamma/IL-10 pattern that correlates with protection. J Immunol (2007) 179:3161-70.

28. Sporri R, Reis e Sousa C. Inflammatory mediators are insufficient for full dendritic cell activation and promote expansion of CD4+ T cell populations lacking helper function. Nat Immunol (2005) 6:163-70. doi:10.1038/ni1162

29. Foulds KE, Wu CY, Seder RA. Th1 memory: implications for vaccine development. Immunol Rev (2006) 211:58-66. doi:10.1111/j.0105-2896.2006.00400.x

30. Streit JA, Recker TJ, Filho FG, Beverley SM, Wilson ME. Protective immunity against the protozoan Leishmania chagasi is induced by subclinical cutaneous infection with virulent but not avirulent organisms. J Immunol (2001) 166:1921-9.

31. Segovia M, Artero JM, Mellado E, Chance ML. Effects of long-term in vitro cultivation on the virulence of cloned lines of Leishmania major promastigotes. Ann Trop Med Parasitol (1992) 86:347-54.

32. de Souza MC, De Assis EA, Gomes RS, Marques Da Silva Ede A, Melo MN, Fietto JL, et al. The influence of ecto-nucleotidases on Leishmania amazonensis infection and immune response in C57B/6 mice. Acta Trop (2010) 115:262-9. doi:10.1016/j.actatropica.2010.04.007

33. Alexander J. A radioattenuated Leishmania major vaccine markedly increases the resistance of CBA mice to subsequent infection with Leishmania mexicana mexicana. Trans R Soc Trop Med Hyg (1982) 76:646-9. doi:10.1016/0035-9203(82) 90232-2

34. Daneshvar H, Hagan P, Phillips RS. Leishmania mexicana H-line attenuated under pressure of gentamicin, potentiates a Thl response and control of cutaneous leishmaniasis in BALB/c mice. Parasite Immunol (2003) 25:589-96. doi:10.1111/j.0141-9838.2004.00671.x

35. Daneshvar H, Burchmore R, Hagan P, Phillips RS. Leishmania major H-line attenuated under pressure of gentamicin, induces a Th1 response which protects susceptible BALB/c mice against infection with virulent L. major. Parasitology (2009) 136:1243-50. doi:10.1017/S0031182009990679

36. Daneshvar H, Molaei MM, Afshar RM, Kamiabi H, Burchmore R, Hagan P, et al. Gentamicin-attenuated Leishmania infantum: a clinicopathological study in dogs. Vet Immunol Immunopathol (2009) 129:28-35. doi:10.1016/j.vetimm. 2008.12.002

37. Daneshvar H, Molaei M, Kamiabi H, Burchmore R, Hagan P, Stephen Phillips R. Gentamicin-attenuated Leishmania infantum: cellular immunity production and protection of dogs against experimental canine leishmaniasis. Parasite Immunol (2010) 32:722-30. doi:10.1111/j.1365-3024.2010.01237.x

38. Daneshvar H, Sedghy F, Dabiri S, Kamiabi H, Molaei MM, Phillips S, et al. Alteration in mononuclear cell subpopulations in dogs immunized with gentamicinattenuated Leishmania infantum. Parasitology (2012) 139:1689-96. doi:10.1017/ S0031182012001187

39. Amaral VF, Teva A, Oliveira-Neto MP, Silva AJ, Pereira MS, Cupolillo E, et al. Study of the safety, immunogenicity and efficacy of attenuated and killed Leishmania (Leishmania) major vaccines in a rhesus monkey (Macaca mulatta) model of the human disease. Mem Inst Oswaldo Cruz (2002) 97:1041-8. doi:10.1590/S0074-02762002000700019

40. Saravia NG, Escorcia B, Osorio Y, Valderrama L, Brooks D, Arteaga L, et al. Pathogenicity and protective immunogenicity of cysteine proteinase-deficient mutants of Leishmania mexicana in non-murine models. Vaccine (2006) 24:4247-59. doi:10.1016/j.vaccine.2005.05.045

41. Kedzierski L, Curtis JM, Doherty PC, Handman E, Kedzierska K. Decreased IL10 and IL-13 production and increased CD44hi T cell recruitment contribute to Leishmania major immunity induced by non-persistent parasites. Eur J Immunol (2008) 38:3090-100. doi:10.1002/eji.200838423

42. Selvapandiyan A, Dey R, Nylen S, Duncan R, Sacks D, Nakhasi HL. Intracellular replication-deficient Leishmania donovani induces long lasting protective immunity against visceral leishmaniasis. J Immunol (2009) 183:1813-20. doi: 10.4049/jimmunol.0900276

43. Carrion J, Folgueira C, Soto M, Fresno M, Requena JM. Leishmania infantum HSP70-II null mutant as candidate vaccine against leishmaniasis: a preliminary evaluation. Parasit Vectors (2011) 4:150. doi:10.1186/1756-3305-4-150

44. Dey R, Dagur PK, Selvapandiyan A, Mccoy JP, Salotra P, Duncan R, et al. Live attenuated Leishmania donovani p27 gene knockout parasites are nonpathogenic and elicit long-term protective immunity in BALB/c mice. J Immunol (2013) 190:2138-49. doi:10.4049/jimmunol.1202801

45. Fiuza JA, Santiago Hda C, Selvapandiyan A, Gannavaram S, Ricci ND, Bueno LL, et al. Induction of immunogenicity by live attenuated Leishmania donovani centrin deleted parasites in dogs. Vaccine (2013) 31:1785-92. doi:10.1016/j.vaccine. 2013.01.048

46. Nolan TJ, Herman R. Effects of long-term in vitro cultivation on Leishmania donovani promastigotes. J Protozool (1985) 32:70-5. doi:10.1111/j.1550-7408. 1985.tb03015.x

47. Lemma A, Cole L. Leishmania enrietti: radiation effects and evaluation of radioattenuated organisms for vaccination. Exp Parasitol (1974) 35:161-9. doi:10.1016/0014-4894(74)90019-8

48. Mutiso JM, Macharia JC, Kiio MN, Ichagichu JM, Rikoi H, Gicheru MM. Development of Leishmania vaccines: predicting the future from past and present experience. J Biomed Res (2013) 27:85-102. doi:10.7555/JBR.27.20120064

49. Selvapandiyan A, Duncan R, Debrabant A, Lee N, Sreenivas G, Nakhasi HL. Genetically modified live attenuated parasites as vaccines for leishmaniasis. Indian J Med Res (2006) 123:455-66.

50. Evans KJ, Kedzierski L. Development of vaccines against visceral leishmaniasis. J Trop Med (2012) 2012:892817. doi:10.1155/2012/892817

51. Selvapandiyan A, Dey R, Gannavaram S, Lakhal-Naouar I, Duncan R, Salotra $\mathrm{P}$, et al. Immunity to visceral leishmaniasis using genetically defined liveattenuated parasites. Re Dai Yi Xue Za Zhi (2012) 2012:631460. doi:10.1155/ 2012/631460

52. Silvestre R, Cordeiro-Da-Silva A, Ouaissi A. Live attenuated Leishmania vaccines: a potential strategic alternative. Arch Immunol Ther Exp (Warsz) (2008) 56:123-6. doi:10.1007/s00005-008-0010-9

53. Mukhopadhyay S, Sen P, Bhattacharyya S, Majumdar S, Roy S. Immunoprophylaxis and immunotherapy against experimental visceral leishmaniasis. Vaccine (1999) 17:291-300. doi:10.1016/S0264-410X(98)90017-2

54. Papadopoulou B, Dumas C. Parameters controlling the rate of gene targeting frequency in the protozoan parasite Leishmania. Nucleic Acids Res (1997) 25:4278-86. doi:10.1093/nar/25.21.4278

55. Yang DM, Fairweather N, Button LL, Mcmaster WR, Kahl LP, Liew FY. Oral Salmonella typhimurium (AroA-) vaccine expressing a major leishmanial surface protein (gp63) preferentially induces T helper 1 cells and protective immunity against leishmaniasis. J Immunol (1990) 145:2281-5.

56. Xu D, Mcsorley SJ, Chatfield SN, Dougan G, Liew FY. Protection against Leishmania major infection in genetically susceptible BALB/c mice by gp63 delivered orally in attenuated Salmonella typhimurium (AroA- AroD-). Immunology (1995) 85:1-7.

57. Xu D, Mcsorley SJ, Tetley L, Chatfield S, Dougan G, Chan WL, et al. Protective effect on Leishmania major infection of migration inhibitory factor, TNF-alpha, and IFN-gamma administered orally via attenuated Salmonella typhimurium. J Immunol (1998) 160:1285-9.

58. Gonzalez CR, Noriega FR, Huerta S, Santiago A, Vega M, Paniagua J, et al. Immunogenicity of a Salmonella typhi CVD 908 candidate vaccine strain expressing the major surface protein gp63 of Leishmania mexicana mexicana. Vaccine (1998) 16:1043-52. doi:10.1016/S0264-410X(97)00267-3

59. Lange UG, Mastroeni P, Blackwell JM, Stober CB. DNA-salmonella enterica serovar Typhimurium primer-booster vaccination biases towards $\mathrm{T}$ helper 1 responses and enhances protection against Leishmania major infection in mice. Infect Immun (2004) 72:4924-8. doi:10.1128/IAI.72.8.4924-4928.2004

60. Schroeder J, Brown N, Kaye P, Aebischer T. Single dose novel Salmonella vaccine enhances resistance against visceralizing $L$. major and $L$. donovani infection in susceptible BALB/c mice. PLoS Negl Trop Dis (2011) 5:e1406. doi:10.1371/journal.pntd.0001406

61. Yam KK, Hugentobler F, Pouliot P, Stern AM, Lalande JD, Matlashewski G, et al. Generation and evaluation of A2-expressing Lactococcus lactis live vaccines against Leishmania donovani in BALB/c mice. J Med Microbiol (2011) 60:1248-60. doi:10.1099/jmm.0.029959-0 
62. Hugentobler F, Di Roberto RB, Gillard J, Cousineau B. Oral immunization using live Lactococcus lactis co-expressing LACK and IL-12 protects BALB/c mice against Leishmania major infection. Vaccine (2012) 30:5726-32. doi:10.1016/ j.vaccine.2012.07.004

63. Hugentobler F, Yam KK, Gillard J, Mahbuba R, Olivier M, Cousineau B. Immunization against Leishmania major infection using LACK- and IL-12-expressing Lactococcus lactis induces delay in footpad swelling. PLoS One (2012) 7:e30945. doi:10.1371/journal.pone.0030945

64. Mizbani A, Taheri T, Zahedifard F, Taslimi Y, Azizi H, Azadmanesh K, et al. Recombinant Leishmania tarentolae expressing the A2 virulence gene as a novel candidate vaccine against visceral leishmaniasis. Vaccine (2009) 28:53-62. doi:10.1016/j.vaccine.2009.09.114

65. Saljoughian N, Taheri T, Zahedifard F, Taslimi Y, Doustdari F, Bolhassani A, et al. Development of novel prime-boost strategies based on a tri-gene fusion recombinant $L$. tarentolae vaccine against experimental murine visceral Leishmaniasis. PLoS Negl Trop Dis (2013) 7:e2174. doi:10.1371/journal.pntd.0002174

66. Dougan G, Hormaeche CE, Maskell DJ. Live oral Salmonella vaccines: potential use of attenuated strains as carriers of heterologous antigens to the immune system. Parasite Immunol (1987) 9:151-60. doi:10.1111/j.1365-3024.1987. tb00496.x

67. Bumann D, Hueck C, Aebischer T, Meyer TF. Recombinant live Salmonella spp. for human vaccination against heterologous pathogens. FEMS Immunol Med Micro (2000) 27:357-64. doi:10.1111/j.1574-695X.2000.tb01450.x

68. Hormaeche CE. Live attenuated Salmonella vaccines and their potential as oral combined vaccines carrying heterologous antigens. J Immunol Methods (1991) 142:113-20. doi:10.1016/0022-1759(91)90298-T

69. Casalta E, Montel MC. Safety assessment of dairy microorganisms: the Lactococcus genus. Int J Food Microbiol (2008) 126:271-3. doi:10.1016/j.ijfoodmicro. 2007.08.013

70. FDA. (1995). Available from: http://www.accessdata.fda.gov/scripts/cdrh/ cfdocs/cfcfr/CFRSearch.cfm?fr=184.1985

71. Bahey-El-Din M, Gahan CG, Griffin BT. Lactococcus lactis as a cell factory for delivery of therapeutic proteins. Curr Gene Ther (2010) 10:34-45. doi: 10.2174/156652310790945557

72. Taylor VM, Munoz DL, Cedeno DL, Velez ID, Jones MA, Robledo SM. Leishmania tarentolae: utility as an in vitro model for screening of antileishmanial agents. Exp Parasitol (2010) 126:471-5. doi:10.1016/j.exppara.2010.05.016

73. Raymond F, Boisvert S, Roy G, Ritt JF, Legare D, Isnard A, et al. Genome sequencing of the lizard parasite Leishmania tarentolae reveals loss of genes associated to the intracellular stage of human pathogenic species. Nucleic Acids Res (2012) 40:1131-47. doi:10.1093/nar/gkr834

74. Azizi H, Hassani K, Taslimi Y, Najafabadi HS, Papadopoulou B, Rafati S. Searching for virulence factors in the non-pathogenic parasite to humans Leishmania tarentolae. Parasitology (2009) 136:723-35. doi:10.1017/S0031182009005873
75. Breton M, Zhao C, Ouellette M, Tremblay MJ, Papadopoulou B. A recombinant non-pathogenic Leishmania vaccine expressing human immunodeficiency virus 1 (HIV-1) Gag elicits cell-mediated immunity in mice and decreases HIV1 replication in human tonsillar tissue following exposure to HIV-1 infection. J Gen Virol (2007) 88:217-25. doi:10.1099/vir.0.81995-0

76. Salehi M, Taheri T, Mohit E, Zahedifard F, Seyed N, Taslimi Y, et al. Recombinant Leishmania tarentolae encoding the HPV type $16 \mathrm{E} 7$ gene in tumor mice model. Immunotherapy (2012) 4:1107-20. doi:10.2217/imt.12.110

77. Okwor I, Uzonna J. Persistent parasites and immunologic memory in cutaneous leishmaniasis: implications for vaccine designs and vaccination strategies. Immunol Res (2008) 41:123-36. doi:10.1007/s12026-008-8016-2

78. Okwor I, Mou Z, Liu D, Uzonna J. Protective immunity and vaccination against cutaneous leishmaniasis. Front Immunol (2012) 3:128. doi:10.3389/fimmu.2012. 00128

79. Sallusto F, Lenig D, Forster R, Lipp M, Lanzavecchia A. Two subsets of memory T lymphocytes with distinct homing potentials and effector functions. Nature (1999) 401:708-12. doi:10.1038/44385

80. Zaph C, Uzonna J, Beverley SM, Scott P. Central memory T cells mediate longterm immunity to Leishmania major in the absence of persistent parasites. Nat Med (2004) 10:1104-10. doi:10.1038/nm1108

81. Pakpour N, Zaph C, Scott P. The central memory CD4+ T cell population generated during Leishmania major infection requires IL-12 to produce IFN-gamma. J Immunol (2008) 180:8299-305.

82. Colpitts S, Scott P. Memory T-cell subsets in parasitic infections. In: Zanetti M, Schoenberger SP, editors. Memory T Cells. New York: Springer (2010). p 145-54.

Conflict of Interest Statement: The authors declare that the research was conducted in the absence of any commercial or financial relationships that could be construed as a potential conflict of interest.

Received: 05 January 2014; paper pending published: 05 February 2014; accepted: 17 March 2014; published online: 31 March 2014.

Citation: Saljoughian N, Taheri T and Rafati S (2014) Live vaccination tactics: possible approaches for controlling visceral leishmaniasis. Front. Immunol. 5:134. doi: 10.3389/fimmu.2014.00134

This article was submitted to Immunotherapies and Vaccines, a section of the journal Frontiers in Immunology.

Copyright (C) 2014 Saljoughian, Taheri and Rafati. This is an open-access article distributed under the terms of the Creative Commons Attribution License (CC BY). The use, distribution or reproduction in other forums is permitted, provided the original author(s) or licensor are credited and that the original publication in this journal is cited, in accordance with accepted academic practice. No use, distribution or reproduction is permitted which does not comply with these terms. 